\title{
Model-based optimization for acoustic characterization of thin hidden layers
}

\author{
Sebastian Wöckel, Hendrik Arndt \\ ifak - Institut für Automation und Kommunikation e.V., \\ Werner-Heisenbergstr. 1, 39106 Magdeburg (Germany), \\ sebastian.woeckel@ifak.eu
}

\begin{abstract}
Summary:
This contribution discusses a model-based optimization method to identify hidden layers and estimate selected material properties gathered from acoustic data. The procedure is exemplified by non-contact air-coupled ultrasound inspections. The method implies a model fitting of the sound propagation in the multi layered media and a derivation of media properties with a genetic algorithm. The modelling and the estimation technique are evaluated with measurement and simulation data of ideal stacked solid and fluid layers, which are "thin" $(<1 \ldots 10 \mathrm{~mm})$ in relation to the acoustic wavelength $(>5 \mathrm{~mm})$.
\end{abstract}

Keywords: ultrasound inspection, parameter estimation, material characterization

\section{Motivation}

The non-destructive physical evaluation of hidden in-between (mostly adhesive) layers is a sophisticated task. Physical parameters of the thin adhesive layer, including its thickness, material distribution, density and elasticity, determine the stability and durability of the assembly. Already, there exists a variety of measurement system that are used for multi-layer inspection. Some apply high frequency electromagnetic signals in the $\mathrm{THz}$-range [1] or high frequency ultrasound to identify stacked layers in size above the wavelength of the corresponding signal. This contribution concerns the common inspection technique based on ultrasound in conjunction with a modelbased algorithm to separate single layers reflections out of the signal. Depending on the application a direct contact, immersion or air-coupled techniques are applied. Although the first provides the highest signal-to-noise ratio and resolution, it is limited to point-selective measurements. The second water-coupled technique provides an automatic scanning, keeping the SNR and resolution, whereat the construction component needs to be immersed in a waterbed. The inspection with air-coupled ultrasound provides the highest degree of freedom - concerning the technical realization. Its main drawbacks are the low defect sensitivity caused by the high reflection loss at the air-solid interface (power balance $P_{\text {out }} / P_{\text {in }} \sim 10^{5}$ ) and the low resolution due to the wavelength accordingly frequency ranges $(<1 \mathrm{MHz})$.

In this context, a signal-oriented method is discussed to overcome the mentioned drawbacks of e.g. air-coupled inspections. The idea bases on the fast estimation of the sound propagation in the multi layered media [2] including an automated derivation of acoustic media properties (mainly density, sound velocity, effective damping and elasticity) by using a mathematical optimization algorithm like a genetic algorithm. The modelling and the estimation technique are exemplified on simulation and empirical data of multiple stacked solid and elastic (adhesive) layers, which are "thin" $(<1 \mathrm{~mm})$ in relation to the acoustic wavelength $(>5 \mathrm{~mm}$ ). This contribution focuses on the algorithm itself exemplified on the estimation of the parameters of a hidden layer, using a simple laboratory measurement setup. This measurement rig provides the ultrasonic air coupled transmission $\left(S_{21}\right)$ or reflection $\left(S_{11}\right)$ measurement on a planar multilayer structure. The thicknesses of the plates, the in-between layer as well as the transducers distances can be adjusted in steps of $\Delta h=100 \mu \mathrm{m}$. For continuous variation of $\left(\rho_{1}, c_{1}, h_{1}\right)$ the hidden layer forms an open cavity filled with a viscous liquid.

The main outcome is an algorithmic method, which delivers supplemental information by a complete acoustic characterization of the medium.

\section{Method: linear propagation model}

The sound propagation through a system of layers can be modelled as a two-port network which is characterized by its transfer $\left(S_{21}\right)$ and reflection $\left(S_{11}\right)$ functions. In time-domain the relative distances and the amplitudes contain the information on the velocity $\left(c_{i}\right)$ and damping $\left(\alpha_{i}\right)$ within each layer and the reflection and acoustic contrast $\left(\rho i, c_{i}\right)$ at each interface. To extract that information a Mason-graph is used, which converts the sound propagation to a deterministic 
two port network. Such graph delivers an analytic expression for the transient wave at each knot of the graph, including all multiple propagation paths, reflections due to acoustic contrasts $(\rho, c)$ and delays $(\Delta t)$ caused by different sound velocity $(c)$. The analytic form of the transfer function $S_{21}$ of a 3-layer-system (Fig. 1) including the transmission within the layers $\left(T_{\mathrm{i}}\right)$ and the reflection at the interfaces $\left(R_{\mathrm{i}}=\Gamma_{\mathrm{i}}\right)$ can be expressed by:

$$
\begin{aligned}
& S_{21}=\frac{1}{\Delta} T_{1} T_{5} \prod_{i=1}^{4} \sqrt{1-R_{i}^{2}} T_{i+1} \\
& \Delta=1+T_{2}^{2} R_{1} R_{2}+T_{3}^{2} R_{2} R_{3}+T_{4}^{2} R_{3} R_{4}+T_{2}^{2} T_{3}^{2} R_{1} R_{3} \\
& +T_{3}^{2} T_{4}^{2} R_{2} R_{4}+T_{2}^{2} T_{3}^{2} T_{4}^{2} R_{1} R_{4} \\
& +T_{2}^{2} T_{4}^{2} R_{1} R_{2} R_{3} R_{4}
\end{aligned}
$$

The main advantage of such an analytic expression is the higher processing speed in an iterative optimization procedure instead of a simulation.

\section{Layer Peeling with Parameter Estimation}

For adapting the model parameters $p$ to the measurement $y_{\text {meas }}$ a minimization of the costfunction $f$ calculating the residual $r(p)$ is applied. The residuals combine the deviation between the model $y_{i}(p)$ and the measurement $y_{\text {meas }}$ by using the difference of the absolute values (signal envelopes via Hilbert-transform) according to the first order Laplace-formulation:

$$
\begin{aligned}
& \min f_{L 1}=\min \|r(\widetilde{\boldsymbol{p}})\|_{1}= \\
& \min \sum_{i=1}^{m}\left|\tilde{y}_{i}(\widetilde{\boldsymbol{p}})-y_{\text {Meas }, i}\right| \\
& f_{L 1}(\rho, c, \alpha)=\sum\left\|\begin{array}{c}
\log \left[\| \text { Hilbert }\left(y_{\text {Model }}\right) \|\right] \\
-\log \left[\left\|\operatorname{Hilbert}\left(y_{\text {Meas }}\right)\right\|\right]
\end{array}\right\|
\end{aligned}
$$

The logarithmic version of the first order norm delivers a smooth curve for prediction of the global minima and a steep decent in the region of the minimum itself. The latter supports an effective and fast gradient optimization. The parameter estimation is divided in two consecutive steps using a differential evolution algorithm for predicting the starting values $p_{0}$ [3] and a gradient based algorithm calculating the material parameters $p$.

\section{Conclusion}

The model equation exemplified here (1) is adequate for a perpendicular incidence only. The accuracy of the 1D-Model estimation mainly depends on the mechanical setup of the measurement the radial symmetry of the transducers and the angular deviation of the incident acoustic field. According to the idealized transmission function for an angular incident acoustic field [4] the error for the layer thickness, the sound velocity and the density of the hidden (unknown) layer will be in size of $5 \%$ for each $1^{\circ}$ deviation of the incident angle. In laboratory measurements and according to the method above, the thickness of the unknown hidden layer can be estimated with an absolute deviation of $5 \%$. The corresponding error for the density and damping coefficient are $<10 \%$ and $>20 \%$ respectively, mainly due to the error propagation. By the help of empiric data as well as 2D-simulations, the performance of the algorithm was evaluated (Fig. 2). According to the bandwidth of the transducers $(B=90 \mathrm{kHz})$ only a reduced region along the theoretical trace of the transmission function $\mathrm{S}_{21}$ (white dotted line) can be used for the calculation. However, even in that case, such algorithm delivers quantitative values of the hidden material and the thickness of the layer.

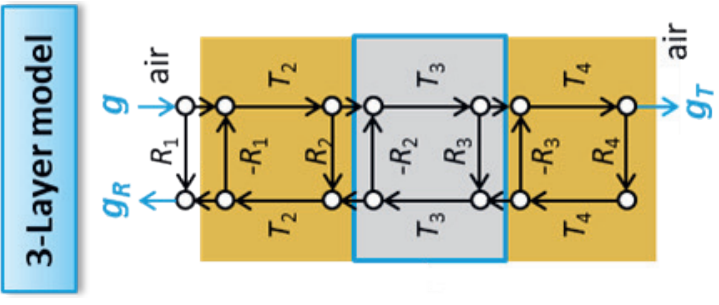

Fig. 1. Mason-Graph of a 3-layer-system; T-transmission function, $R$ - reflection function, $g$ - signal

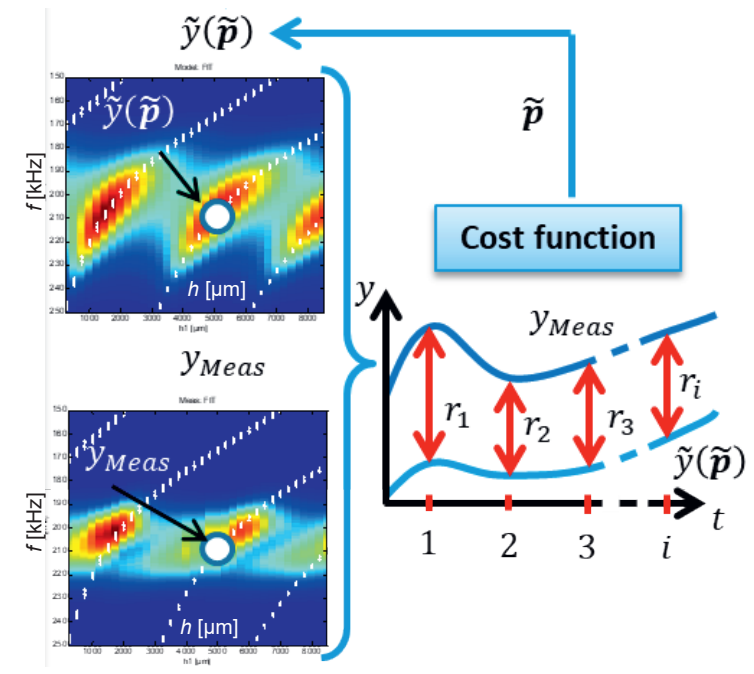

Fig. 2. Schematic for the optimization of the model function $y(p)$ and the Measurement $y_{\text {Meas in frequency }}$ domain for an ultrasonic transmission on a 3-layersystem (Acrylic (6 mm) - Water ( $h$ - variable) - Acrylic (6 mm)) with burst-excitation of $(207.5 \mathrm{kHz}+/-45 \mathrm{kHz})$.

\section{References}

[1] J. Jonuscheit, et.al 'Contact-Free Multilayer Thickness Determination of Industrial Coatings using THz Measuring Techniques', TM 2/2012.

[2] F. Hägglund, et.al 'Model-based estimation of thin multi-layered media using ultrasonic measurements', IEEE UFFC, vol.56, no.8, 2009.

[3] J. E. Carlson, 'Characterization of thin layers in multi-layered structures: on the problem of finding starting values for numerical solutions to inverse problems', in 2009 IUS: Italy, 2009.

[4] Rose, Ultrasonic waves in solid media, 2008 